Proyecciones Journal of Mathematics

Vol. 30, No 3, pp. 401-413, December 2011.

Universidad Católica del Norte

Antofagasta - Chile

\title{
An extension of the skew-generalized normal distribution and its derivation
}

\author{
OSVALDO VENEGAS \\ UNIVERSIDAD CATÓLICA DE TEMUCO, CHILE \\ ANTONIO I. SANHUEZA \\ UNIVERSIDAD DE LA FRONTERA, CHILE \\ and \\ HÉCTOR W. GÓMEZ \\ UNIVERSIDAD DE ANTOFAGASTA, CHILE \\ Received : October 2010. Accepted : September 2011
}

\begin{abstract}
In this paper, we introduce a new class of skew-symmetric distributions which are formulated based on cumulative distributions of skew-symmetric densities. This new class is an extension of other skew-symmetric distributions that have already been studied. We give special attention to a family from this class that could be seen as an extension of the skew-generalized-normal model introduced by ArellanoValle et al.(2004). We study the main properties, stochastic representation, moments and an extension of this new model.
\end{abstract}

Key Words : Asymmetry; Skew-Generalized Normal Distribution; Skew-Normal Distribution. 


\section{Introduction}

It is known that the normal distribution is very useful in different applications in statistics. However, it is also known that this distribution is not appropriate in those cases where symmetry is absent. In this respect the skew-normal family has been studied among others by Azzalini (1985), Henze (1986), Pewsey (2000) and Martinez et al. (2008), and reviewed in the book by Genton (2004). The univariate skew-normal model has the following density function:

$$
\phi_{\lambda}(x, \mu, \sigma)=\frac{2}{\sigma} \phi\left(\frac{x-\mu}{\sigma}\right) \Phi\left(\lambda \frac{x-\mu}{\sigma}\right), x \in \mathbf{R}, \lambda \in \mathbf{R}, \mu \in \mathbf{R}, \sigma>0,
$$

where $\phi(x)$ and $\Phi(x)$ denote the density function and cumulative distribution function of the standard normal distribution, respectively. We denote the distribution of Azzalini as $X \sim S N(\mu, \sigma, \lambda)$. A better family of distributions that shows a better behavior, particularly on the side with smaller mass, than the skew-normal distribution is the Skew-Generalized Normal (SGN) Distribution introduced by Arellano-Valle et al. (2004), which has the following density function:

$$
\begin{aligned}
& \phi_{\lambda_{1}, \lambda_{2}}(x)=\frac{2}{\sigma} \phi\left(\frac{x-\mu}{\sigma}\right) \Phi\left(\frac{\lambda_{1}(x-\mu)}{\sqrt{\sigma^{2}+\lambda_{2}(x-\mu)^{2}}}\right), x \in \mathbf{R}, \lambda_{1} \in \mathbf{R}, \lambda_{2} \geq 0, \\
& \mu \in \mathbf{R}, \sigma>0 .
\end{aligned}
$$

We denote this distribution by $X \sim S G N\left(\mu, \sigma, \lambda_{1}, \lambda_{2}\right)$.

The main objective of this paper is to introduce a new class of asymmetric distributions that contain an unlimited number of skew-symmetric models. We give special attention to a skew model that is an extension of the skew-generalized normal distribution and the skew normal distribution. In Section 2, we define this new extension and call it the Extended Skew Generalized Normal Distribution (ESGN), and also study its basic properties. In Section 3, we develop important probabilistic properties of the new 
family of distribution, including the stochastic representation. In Section 4 , we obtain the moments of the random variable with ESGN distribution and finally, Section 5 deals with a location-scale extension of the ESGN distribution and derives a generalization of the ESGN distribution.

\section{A Class of Skew Distribution}

The following Lemma was presented by Azzalini (2005), and is a fundamental result for generating skew-symmetric distributions.

Lemma 1. Let $g$ be a probability density function symmetric about zero, and $G$ is a distribution function such that $G^{\prime}$ there exist and is a density function symmetric about zero, then

$$
f(x)=2 g(x) G(w(x)), \quad-\infty<x<\infty,
$$

is a density function for any odd function $w(\cdot)$.

Some subclass of skew-symmetric distributions generated from this lemma have been studied among others by Gupta et al. (2002), Nadarajah and Kotz (2003) and Gómez et al. (2007). In the following proposition we introduce a new subclass of skew-symmetric models.

Proposition 1. Let $g$ be a probability density function symmetric about zero, and $H_{n(x)}$ be a distribution function, from a skew density function, of the form $H_{n(x)}(m(x))=\int_{-\infty}^{m(x)} h(t) d t$, with $h(t)=2 g(t) G(n(x) t)$ as defined in (3) where $n(x)$ is any odd function, then

$$
\varphi(x)=2 g(x) H_{n(x)}(m(x)), \quad-\infty<x<\infty,
$$

is a density function for any odd function $m(\cdot)$.

Proof: Let $R(x)=H_{n(x)}(m(x))=\int_{-\infty}^{m(x)} 2 g(t) G(n(x) t) d t$.

Now, since $n(x)$ and $m(x)$ are odd functions, we have

$$
R(-x)=\int_{-\infty}^{m(-x)} 2 g(t) G(n(-x) t) d t=\int_{m(x)}^{\infty} 2 g(t) G(n(x) t) d t .
$$

Then $R(x)+R(-x)=1$ and $R^{\prime}(-x)=R^{\prime}(x)$, which shows that $R^{\prime}$ is symmetric about zero. Thus, by using Lemma 1 we prove this proposition. 
Example 1. If we consider $H_{n(x)}(m(x))$ to be an extension of the cumulative distribution function from the symmetric density function $g$, then we obtain an extension of the family studied by Gupta et al. (2002).

Example 2. If we consider $H_{n(x)}(m(x))$ to be the cumulative distribution function of a skew-symmetric density and $g=\phi$, then we obtain an extension of the family studied by Nadarajah and Kotz (2003).

Example 3. If we consider $H_{n(x)}(m(x))=\Phi_{n(x)}(m(x))$ and $g$ to be any symmetric density function, then we obtain an extension of the family studied by Gómez et al. (2007).

The following example is the main interest of this paper and we present it as a definition.

Definition 1. A random variable $X$ is said to have a Extended Skew Generalized Normal Distribution if its density function is given by

$\phi_{\lambda_{1}, \lambda_{2}, \lambda}(x)=2 \phi(x) \Phi_{\mu\left(x, \lambda_{2}, \lambda\right)}\left(\frac{\lambda_{1} x}{\sqrt{1+\lambda_{2} x^{2}}}\right), x \in \mathbf{R}, \lambda_{1} \in \mathbf{R}, \lambda_{2} \geq 0, \lambda \in \mathbf{R}$,

where

$$
\Phi_{\mu\left(x, \lambda_{2}, \lambda\right)}\left(\frac{\lambda_{1} x}{\sqrt{1+\lambda_{2} x^{2}}}\right)=\int_{-\infty}^{\frac{\lambda_{1} x}{\sqrt{1+\lambda_{2} x^{2}}}} 2 \phi(t) \Phi\left(\mu\left(x, \lambda_{2}, \lambda\right) t\right) d t
$$

and

$$
\mu\left(x, \lambda_{2}, \lambda\right)=\frac{-\sqrt{\lambda_{2}} \lambda x}{\sqrt{1+\lambda_{2} x^{2}+\lambda^{2}}} .
$$

We denote the Extended Skew Generalized Normal Distribution as $X \sim$ $\operatorname{ESGN}\left(\lambda_{1}, \lambda_{2}, \lambda\right)$.

Remark 1. 1. Let it be considered that $g=\phi, H=\Phi, n(x)=\mu\left(x, \lambda_{2}, \lambda\right)$, $m(x)=\frac{\lambda_{1} x}{\sqrt{1+\lambda_{2} x^{2}}}$ and by applying Proposition 1 we can prove that (5) is a density function.

2. We can also prove that (5) is a density function by using the result shown by Chiogna (1998), since if $Z \sim S N(\lambda)$, then the random variable $Y=\Phi(h Z+k)$ has first moment: 


$$
E_{\lambda}[\Phi(h Z+k)]=\Phi_{\mu(h, \lambda)}\left(k / \sqrt{1+h^{2}}\right) \text {, with } \mu(h, \lambda)=-\frac{h \lambda}{\sqrt{1+h^{2}+\lambda^{2}}} .
$$

We show several of the possible shapes obtained from (5) under various choices of $\left(\lambda_{1}, \lambda_{2}, \lambda\right)$.

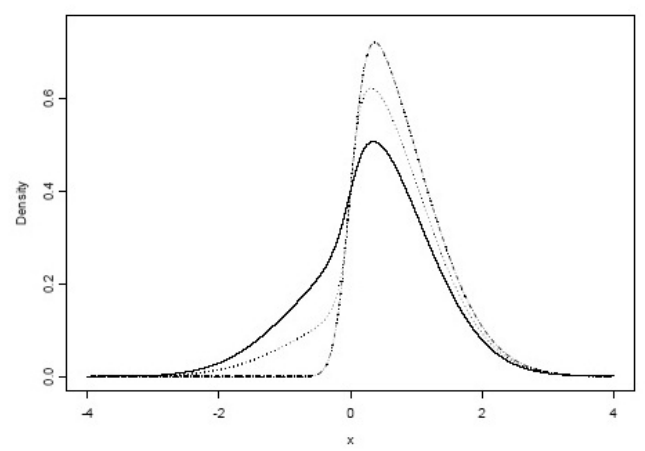

Figure 1 : The $\operatorname{ESGN}(5,20,-2)$ (solid line), $\operatorname{SGN}(5,20)$ (dotted line) and $S N(5)$ (dashed line) densities.

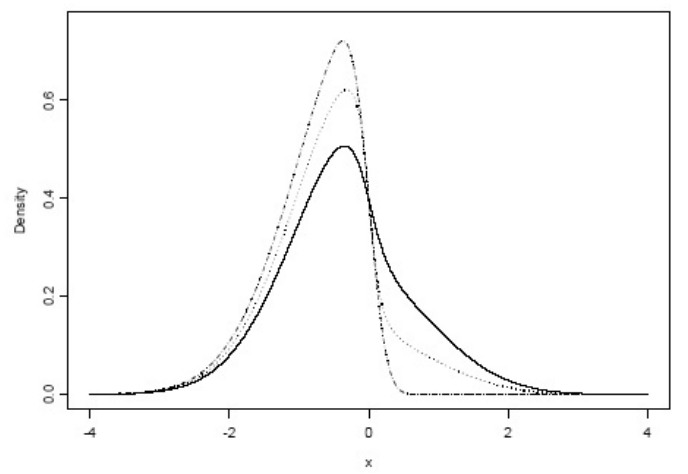

Figure 2 : The $\operatorname{ESGN}(-5,20,2)$ (solid line), $\operatorname{SGN}(-5,20)$ (dotted line) and $S N(-5)$ (dashed line) densities.

Remark 2. It should be noted that the mechanism of asymmetry in our model, is presented by the joint action of the parameters $\lambda_{1}, \lambda_{2}$ and $\lambda$. Phenomenon already observed in the model SGN introduced by ArellanoValle et al. (2004).

Proposition 2. (a) Let $X$ be a random variable with $X \sim \operatorname{ESGN}\left(\lambda_{1}, \lambda_{2}, \lambda\right)$. Then 
1. $\phi_{\lambda_{1}, \lambda_{2}, \lambda=0}(x)=\phi_{\lambda_{1}, \lambda_{2}}(x)$, i.e. $\operatorname{ESGN}\left(\lambda_{1}, \lambda_{2}, \lambda=0\right)=S G N\left(\lambda_{1}, \lambda_{2}\right)$.

2. $\phi_{\lambda_{1}, \lambda_{2}=0, \lambda}(x)=\phi_{\lambda}(x)$, i.e. $\operatorname{ESGN}\left(\lambda_{1}, \lambda_{2}=0, \lambda\right)=S N\left(\lambda_{1}\right)$.

3. $\phi_{\lambda_{1}=0, \lambda_{2}, \lambda=0}(x)=\phi(x)$, i.e. $\operatorname{ESGN}\left(\lambda_{1}=0, \lambda_{2}, \lambda=0\right)=N(0,1)$.

4. $\phi_{-\lambda_{1}, \lambda_{2},-\lambda}(x)+\phi_{-\lambda_{1}, \lambda_{2},-\lambda}(x)=2 \phi(x), x \in \mathbf{R}$.

5. $-X \sim E S G N\left(-\lambda_{1}, \lambda_{2},-\lambda\right)$.

6. $\lim _{\lambda_{1} \rightarrow+\infty} \phi_{\lambda_{1}, \lambda_{2}, \lambda}(x)=2 \phi(x) I\{x \geq 0\}$ and

$\lim _{\lambda_{1} \rightarrow \infty} \phi_{\lambda_{1}, \lambda_{2}, \lambda}(x)=2 \phi(x) I\{x \leq 0\}$, for all $\lambda_{2}, \lambda$.

7. $\lim _{\lambda_{1} \rightarrow \pm \infty} \phi_{\lambda_{1}, \lambda_{1}^{2}, \lambda}(x)=2 \phi(x) \Phi_{\mu(x, \lambda)}( \pm \operatorname{sgn}(x))$, where $\mu(x, \lambda)=\mp \lambda \operatorname{sgn}(x)$.

(b) Let $\Phi_{\lambda_{1}, \lambda_{2}, \lambda}(x)$ be the CDF of the random variable $X \sim \operatorname{ESGN}\left(\lambda_{1}, \lambda_{2}, \lambda\right)$. Then:

1. $\Phi_{\lambda_{1}, \lambda_{2}, \lambda=0}(x)=\Phi_{\lambda_{1}, \lambda_{2}}(x)$

2. $\Phi_{\lambda_{1}, \lambda_{2}=0, \lambda}(x)=\Phi_{\lambda_{1}}(x)$

3. $\Phi_{\lambda_{1}=0, \lambda_{2}, \lambda=0}(x)=\Phi(x)$

4. $\Phi_{\lambda_{1}, \lambda_{2}, \lambda}(-x)=1-2 \Phi(x)+\Phi_{\lambda_{1}, \lambda_{2}, \lambda}(x)$

Proof: The proofs of the items (a) and (b) are obtained directly by using the functions given in (5) and (6) of Definition 1.

Remark 3. When $\lambda=0$ in part 4 of Proposition 2(b), we have that:

$$
\Phi_{\lambda_{1}, \lambda_{2}, 0}(-x)=1-2 \Phi(x)+\Phi_{\lambda_{1}, \lambda_{2}, 0}(x)=1-\Phi_{-\lambda_{1}, \lambda_{2}}(-x),
$$

where $\Phi_{\lambda_{1}, \lambda_{2}}(x)$ is the CDF of the Skew-Generalized Normal Distribution.

\section{Some Important Properties}

We derive important probabilistic properties from the ESGN distribution. The following two propositions show similar results to those obtained from the SGN distribution, where the distribution of the absolute value of a ESGN distribution is half-normal, and the square of the ESGN distribution is a chi-square with one degree of freedom. The Proposition 4 in this section shows the stochastic representation of the ESGN distribution.

Proposition 3. If $X \sim E S G N\left(\lambda_{1}, \lambda_{2}, \lambda\right)$ and $Y \sim N(0,1)$, then the random variables $|X|$ and $|Y|$ are identically distributed Standard Half-Normal. 
Proof: Let $W=|X|$, then the density function of $W$ is given by:

$$
\begin{aligned}
f_{W}(w) & =\phi_{\lambda_{1}, \lambda_{2}, \lambda}(w)+\phi_{\lambda_{1}, \lambda_{2}, \lambda}(-w) \\
& =2 \phi(w)\left\{\Phi_{\mu\left(w, \lambda_{2}, \lambda\right)}\left(\frac{\lambda_{1} w}{\sqrt{1+\lambda_{2} w^{2}}}\right)+\Phi_{-\mu\left(w, \lambda_{2}, \lambda\right)}\left(\frac{-\lambda_{1} w}{\sqrt{1+\lambda_{2} w^{2}}}\right)\right\} \\
& =2 \phi(w), w>0,
\end{aligned}
$$

which has the same distribution as $|Y|$.

Corollary 1. If $X \sim \operatorname{ESGN}\left(\lambda_{1}, \lambda_{2}, \lambda\right)$, then $Y=X^{2}$ has a chi-square distribution with 1 degree of freedom, i. e. $Y \sim \chi_{1}^{2}$.

Proposition 4. If $X \mid Y=y \sim S N(y)$ and $Y \sim S N\left(\lambda_{1}, \lambda_{2}, \lambda\right)$, then $X \sim$ $\operatorname{ESGN}\left(\lambda_{1}, \lambda_{2}, \lambda\right)$.

\section{Proof:}

$$
\begin{aligned}
f_{X}(x) & =\int_{-\infty}^{\infty} 2 \phi(x) \Phi(y x) \frac{2}{\sqrt{\lambda_{2}}} \phi\left(\frac{y-\lambda_{1}}{\sqrt{\lambda_{2}}}\right) \Phi\left(\lambda\left(\frac{y-\lambda_{1}}{\sqrt{\lambda_{2}}}\right)\right) d y \\
& =2 \phi(x) \int_{-\infty}^{\infty} \Phi\left(x \lambda_{1}+x \sqrt{\lambda_{2}} u\right) 2 \phi(u) \Phi(\lambda u) d u \\
& =2 \phi(x) E_{\lambda}\left\{\Phi\left(x \lambda_{1}+x \sqrt{\lambda_{2}} U\right)\right\} \\
& =2 \phi(x) \Phi_{\mu\left(x, \lambda_{2}, \lambda\right)}\left(\frac{\lambda_{1} x}{\sqrt{1+\lambda_{2} x^{2}}}\right)
\end{aligned}
$$

by using the expression in $(2.6)$, where $\mu\left(x, \lambda_{2}, \lambda\right)=\frac{-\sqrt{\lambda_{2}} \lambda x}{\sqrt{1+\lambda_{2} x^{2}+\lambda^{2}}}$.

\section{Moments}

In this section we obtain the $n$-th moment of the random variable $X \sim$ $\operatorname{ESGN}\left(\lambda_{1}, \lambda_{2}, \lambda\right)$. In the case that $n$ is even, the moments are derived from the chi-square distribution with 1 degree of freedom. When $n$ is odd, we can derive an implicit expression for the $n$-th moment of $X$. In this respect, we denote:

(4.1) $a_{n}\left(\lambda_{1}, \lambda_{2}, \lambda\right)=\int_{0}^{\infty} \frac{u^{n}}{\sqrt{2 \pi}} \exp \left\{-\frac{u}{2}\right\} \Phi_{\mu\left(\sqrt{u}, \lambda_{2}, \lambda\right)}\left(\frac{\lambda_{1} \sqrt{u}}{\sqrt{1+\lambda_{2} u}}\right) d u$ and 


$$
b_{n}\left(\lambda_{1}, \lambda_{2}, \lambda\right)=\int_{0}^{\infty} \frac{u^{n}}{\sqrt{2 \pi}} \exp \left\{-\frac{u}{2}\right\} \Phi_{-\mu\left(\sqrt{u}, \lambda_{2}, \lambda\right)}\left(\frac{\lambda_{1}}{\sqrt{\lambda_{2}}}\right) d u .
$$

We can show that for all $\lambda_{2} \geq 0$ :

$a_{n}\left(0, \lambda_{2}, 0\right)=a_{n}(0,0, \lambda)=\frac{2^{n}}{\sqrt{2 \pi}} \Gamma(n+1)$ and $b_{n}\left(0, \lambda_{2}, 0\right)=\frac{2^{n}}{\sqrt{2 \pi}} \Gamma(n+1)$.

Proposition 5. Let $X$ be a random variable with $X \sim \operatorname{ESGN}\left(\lambda_{1}, \lambda_{2}, \lambda\right)$. Then we have that:

$$
E\left(X^{2 k+1}\right)=2\left\{a_{k}\left(\lambda_{1}, \lambda_{2}, \lambda\right)-a_{k}(0,0, \lambda)\right\}, \quad \text { with } \quad k=0,1,2, \ldots
$$

\section{Proof:}

$$
\begin{aligned}
\mathrm{E}\left(\mathrm{X}^{2 k+1}\right)= & \int_{-\infty}^{\infty} x^{2 k+1} 2 \phi(x) \Phi_{\mu\left(x, \lambda_{2}, \lambda\right)}\left(\frac{\lambda_{1} x}{\sqrt{1+\lambda_{2} x^{2}}}\right) d x \\
= & \int_{0}^{\infty}(-x)^{2 k+1} 2 \phi(x) \Phi_{-\mu\left(x, \lambda_{2}, \lambda\right)}\left(\frac{-\lambda_{1} x}{\sqrt{1+\lambda_{2} x^{2}}}\right) d x+ \\
\int_{0}^{\infty} x^{2 k+1} 2 \phi(x) \Phi_{\mu\left(x, \lambda_{2}, \lambda\right)}\left(\frac{\lambda_{1} x}{\sqrt{1+\lambda_{2} x^{2}}}\right) d x & 2\left[\int_{0}^{\infty} x^{2 k+1} 2 \phi(x) \Phi_{\mu\left(x, \lambda_{2}, \lambda\right)}\left(\frac{\lambda_{1} x}{\sqrt{1+\lambda_{2} x^{2}}}\right) d x\right. \\
= & \left.-\int_{0}^{\infty} x^{2 k+1} \phi(x) d x\right] \\
= & 2\left[a_{k}\left(\lambda_{1}, \lambda_{2}, \lambda\right)-a_{k}(0,0, \lambda)\right] .
\end{aligned}
$$

Lemma 2. For any $\lambda_{1}>0, \lambda_{2}>0, \lambda \in \mathbf{R}$ and $n=0,1,2, \ldots$ we have:

$$
a_{n}\left(0, \lambda_{2}, \lambda\right) \leq a_{n}\left(\lambda_{1}, \lambda_{2}, \lambda\right) \leq 4 a_{n}(0,0, \lambda) \phi\left(\frac{\lambda_{1}}{\sqrt{\lambda_{2}}}\right)-b_{n}\left(\lambda_{1}, \lambda_{2}, \lambda\right),
$$

where $a_{n}\left(\lambda_{1}, \lambda_{2}, \lambda\right)$ and $b_{n}\left(\lambda_{1}, \lambda_{2}, \lambda\right)$ are given in (9) and (10), respectively. Also, if $\lambda>0$, we have

$$
a_{n}(0,0, \lambda) \leq a_{n}\left(0, \lambda_{2}, \lambda\right)
$$

Proof: Using the fact that $\Phi_{\mu\left(\sqrt{u}, \lambda_{2}, \lambda\right)}(t)$ is an increasing function of $t$, we have

$$
\Phi_{\mu\left(\sqrt{u}, \lambda_{2}, \lambda\right)}(0) \leq \Phi_{\mu\left(\sqrt{u}, \lambda_{2}, \lambda\right)}\left(\frac{\lambda_{1}}{\sqrt{1+\lambda_{2} u}}\right) \leq \Phi_{\mu\left(\sqrt{u}, \lambda_{2}, \lambda\right)}\left(\frac{\lambda_{1}}{\sqrt{\lambda_{2}}}\right),
$$
and then: 


$$
\begin{aligned}
& \int_{0}^{\infty} \frac{u^{n}}{\sqrt{2 \pi}} \exp \left\{-\frac{u}{2}\right\} \Phi_{\mu\left(\sqrt{u}, \lambda_{2}, \lambda\right)}(0) d u \\
& \quad \leq \int_{0}^{\infty} \frac{u^{n}}{\sqrt{2 \pi}} \exp \left\{-\frac{u}{2}\right\} \Phi_{\mu\left(\sqrt{u}, \lambda_{2}, \lambda\right)}\left(\frac{\lambda_{1} \sqrt{u}}{\sqrt{1+\lambda_{2} u}}\right) d u \\
& \quad \leq \int_{0}^{\infty} \frac{u^{n}}{\sqrt{2 \pi}} \exp \left\{-\frac{u}{2}\right\} \Phi_{\mu\left(\sqrt{u}, \lambda_{2}, \lambda\right)}\left(\frac{\lambda_{1}}{\sqrt{\lambda_{2}}}\right) d u
\end{aligned}
$$

where

$$
\begin{aligned}
& \int_{0}^{\infty} \frac{u^{n}}{\sqrt{2 \pi}} \exp \left\{-\frac{u}{2}\right\} \Phi_{\mu\left(\sqrt{u}, \lambda_{2}, \lambda\right)}\left(\frac{\lambda_{1}}{\sqrt{\lambda_{2}}}\right) d u \\
& \quad=2 \int_{0}^{\infty} \frac{u^{n}}{\sqrt{2 \pi}} \exp \left\{-\frac{u}{2}\right\} \Phi\left(\frac{\lambda_{1}}{\sqrt{\lambda_{2}}}\right) d u \\
& \quad-\int_{0}^{\infty} \frac{u^{n}}{\sqrt{2 \pi}} \exp \left\{-\frac{u}{2}\right\} \Phi_{-\mu\left(\sqrt{u}, \lambda_{2}, \lambda\right)}\left(\frac{\lambda_{1}}{\sqrt{\lambda_{2}}}\right) d u
\end{aligned}
$$

which shows the first part.

On the other hand, when $\lambda>0$,

$$
\begin{aligned}
\int_{0}^{\infty} \frac{u^{n}}{\sqrt{2 \pi}} e^{-\frac{u}{2}} \Phi_{\mu\left(\sqrt{u}, \lambda_{2}, \lambda\right)}(0) d u \\
\quad=\int_{0}^{\infty} \frac{u^{n}}{\sqrt{2 \pi}} \exp \left\{-\frac{u}{2}\right\}\left(\int_{-\infty}^{0} 2 \phi(t) \Phi\left(\mu\left(\sqrt{u}, \lambda_{2}, \lambda\right) t\right) d t\right) d u \\
\quad \geq \int_{0}^{\infty} \frac{u^{n}}{\sqrt{2 \pi}} \exp \left\{-\frac{u}{2}\right\}\left(\int_{-\infty}^{0} 2 \phi(t) \Phi(0) d t\right) d u \\
\quad=\frac{1}{2} \int_{0}^{\infty} \frac{u^{n}}{\sqrt{2 \pi}} \exp \left\{-\frac{u}{2}\right\} d u,
\end{aligned}
$$

which shows the second part.

Proposition 6. Let $X$ be a random variable with $X \sim \operatorname{ESGN}\left(\lambda_{1}, \lambda_{2}, \lambda\right)$. Then the moment generating function of $X$ is given by:

$$
M_{X}(t)=2 \exp \left\{\frac{t^{2}}{2}\right\} E\left[\Phi_{\mu\left(y+t, \lambda_{2}, \lambda\right)}\left(\frac{\lambda_{1}(Y+t)}{\sqrt{1+\lambda_{2}(Y+t)^{2}}}\right)\right], Y \sim N(0,1)
$$

\section{An Extension Class of Densities}

In this section we extend the definition of (5) to the location-scale family. Also, we introduce a new family of asymmetric normal distributions that contains the Extended Skew Generalized Normal Distribution. 
Definition 2. A random variable $W=\mu+\sigma X$, where $X \sim \operatorname{ESGN}\left(\lambda_{1}, \lambda_{2}, \lambda\right)$ is said to have the Location-Scale Extended Skew Generalized Normal Distribution, with density function given by:

$$
f_{W}(w)=\frac{2}{\sigma} \phi\left(\frac{w-\mu}{\sigma}\right) \Phi_{\mu\left(\frac{w-\mu}{\sigma}, \lambda_{2}, \lambda\right)}\left(\frac{\lambda_{1}(w-\mu)}{\sqrt{\sigma^{2}+\lambda_{2}(w-\mu)^{2}}}\right), w \in \mathbf{R},
$$

$$
\lambda_{1} \in \mathbf{R}, \lambda_{2} \geq 0, \lambda \in \mathbf{R}, \mu \in \mathbf{R}, \sigma>0,
$$

where

and

$$
\Phi_{\mu\left(\frac{w-\mu}{\sigma}, \lambda_{2}, \lambda\right)}\left(\frac{\lambda_{1}(w-\mu)}{\sqrt{\sigma^{2}+\lambda_{2}(w-\mu)^{2}}}\right)
$$

$\mu\left(\frac{w-\mu}{\sigma}, \lambda_{2}, \lambda\right)$ are given in (6) and (7), respectively.

We denote this location-scale distribution by $W \sim \operatorname{ESGN}\left(\mu, \sigma ; \lambda_{1}, \lambda_{2}, \lambda\right)$.

Remark 4. Note that the distribution (11) is a model with five parameters, estimating they can be a problem. But today, due to computing resources, particularly the statistical software, are becoming increasingly efficient, we believe it is necessary support when desired perform this task.

Corollary 2. If $W \mid Y=y \sim S N(\mu, \sigma, y)$ and $Y \sim S N\left(\lambda_{1}, \lambda_{2}, \lambda\right)$, then $W \sim \operatorname{ESGN}\left(\mu, \sigma, \lambda_{1}, \lambda_{2}, \lambda\right)$.

Definition 3. The random variable $X$ with density function

$$
\begin{aligned}
& \qquad \phi_{\lambda_{1}, \lambda_{2}, \lambda, \alpha}(x)=2 \phi(x) \Phi_{\mu\left(x, \lambda_{2}, \lambda, \alpha\right)}\left(\frac{\lambda_{1} x}{\sqrt{1+\left(\alpha+\lambda_{2}\right) x^{2}}}\right), x \in \mathbf{R}, \lambda_{1} \in \mathbf{R}, \\
& \quad \lambda_{2} \geq 0, \lambda \in \mathbf{R}, \alpha \geq 0,(5.2) \\
& \text { where }
\end{aligned}
$$

$\Phi_{\mu\left(x, \lambda_{2}, \lambda, \alpha\right)}\left(\frac{\lambda_{1} x}{\sqrt{1+\left(\alpha+\lambda_{2}\right) x^{2}}}\right)=\int_{-\infty}^{\frac{\lambda_{1} x}{\sqrt{1+\left(\alpha+\lambda_{2}\right) x^{2}}}} 2 \phi(t) \Phi\left(\mu\left(x, \lambda_{2}, \lambda, \alpha\right) t\right) d t$ 
and

$$
\mu\left(x, \lambda_{2}, \lambda, \alpha\right)=\frac{-\sqrt{\lambda_{2}} \lambda x}{\sqrt{1+\left(\lambda_{2}+\alpha\right) x^{2}+\lambda^{2}\left(1+\alpha x^{2}\right)}}
$$

has a Beyond Skew-Generalized Normal (BSGN) Distribution, which is a generalization of the random variable with the density function given in (5). We denote this generalization as $X \sim B S G N\left(\alpha, \lambda_{1}, \lambda_{2}, \lambda\right)$.

Remark 5. 1. We note that for $\alpha=0$ we reproduce the Extended Skew Generalized Normal Distribution.

2. We may consider $g=\phi, H=\Phi, n(x)=\mu\left(x, \alpha, \lambda_{2}, \lambda\right), m(x)=$ $\frac{\lambda_{1} x}{\sqrt{1+\left(\alpha+\lambda_{2}\right) x^{2}}}$ and by applying Proposition 1 we can prove that (12) is a density function.

Proposition 7. If $X \mid Y=y \sim S G N(y, \alpha)$ and $Y \sim S N\left(\lambda_{1}, \lambda_{2}, \lambda\right)$, then $X \sim \operatorname{BSGN}\left(\alpha, \lambda_{1}, \lambda_{2}, \lambda\right)$.

\section{Proof:}

$$
\begin{aligned}
f_{X}(x) & =\int_{-\infty}^{\infty} 2 \phi(x) \Phi\left(\frac{y x}{\sqrt{1+\alpha x^{2}}}\right) \frac{2}{\sqrt{\lambda_{2}}} \phi\left(\frac{y-\lambda_{1}}{\sqrt{\lambda_{2}}}\right) \Phi\left(\lambda\left(\frac{y-\lambda_{1}}{\sqrt{\lambda_{2}}}\right)\right) d y \\
& =2 \phi(x) \int_{-\infty}^{\infty} \Phi\left(\frac{\left(\lambda_{1}+\sqrt{\lambda_{2}} u\right) x}{\sqrt{1+\alpha x^{2}}}\right) 2 \phi(u) \Phi(\lambda u) d u \\
& =2 \phi(x) E_{\lambda}\left\{\Phi\left(\frac{\lambda_{1} x+\sqrt{\lambda_{2}} x U}{\sqrt{1+\alpha x^{2}}}\right)\right\} \\
& =2 \phi(x) \Phi_{\mu\left(x, \alpha, \lambda_{2}, \lambda\right)}\left(\frac{\lambda_{1} x}{\sqrt{1+\left(\alpha+\lambda_{2}\right) x^{2}}}\right),
\end{aligned}
$$

by using the expression in $(2.6)$, where $\mu\left(x, \alpha, \lambda_{2}, \lambda\right)=\frac{-\sqrt{\lambda_{2}} \lambda x}{\sqrt{1+\left(\lambda_{2}+\alpha\right) x^{2}+\lambda^{2}\left(1+\alpha x^{2}\right)}}$.

\section{Acknowledgments}

The authors acknowledge helpful comments and suggestions the referee which substantially improved the presentation. The research of O. Venegas was supported by DGIP 2009|2|1. The research of A. I. Sanhueza was supported by FONDECYT 1090265. The research of H. W. Gómez was supported by FONDECYT 1090411. 


\section{References}

[1] Arellano-Valle, R. B., Gómez, H. W. and Quintana, F. A. A new class of skew-normal distributions. Communications in Statistics : Theory and Methods, 33(7), pp. 1465-1480, (2004).

[2] Azzalini, A. A class of distributions which includes the normal ones. Scandinavian Journal Statistics, 12, pp. 171-178, (1985).

[3] Azzalini, A. The skew-normal distribution and related multivariate families (with discussion). Scandinavian Journal Statistics, 32, pp. 159-188, (2005).

[4] Chiogna, M. Some results on the scalar skew-normal distribution. J. Ital. Statist. Soc., 7, pp. 1-13, (1998).

[5] Genton, M. G., Ed. Skew-elliptical distributions and their applications: a journey beyond normality. Chapman \& Hall/CRC, (2004).

[6] Gómez, H. W., Venegas, O. and Bolfarine H. Skew-symmetric distributions generated by the distribution function of the normal distribution. Environmetrics, 18, pp. 395-407, (2007).

[7] Gupta, A. K., Chang, F. C. and Huang, W. J. Some skew-symmetric model. Randon Operators Stochastic Equations, 10, pp. 133-140, (2002).

[8] Henze, N. A probabilistic representation of the skew-normal distribution. Scandinavian Journal Statistics, 13, pp. 271-275, (1986).

[9] Martinez, E., Varela, H., Gómez, H. W. and Bolfarine, H. A note on the likelihood and moments of the skew-normal distribution. Statistics and Operations Research Transactions (SORT). 32(1), pp. 57-66, (2008).

[10] Nadarajah, S., Kotz, S. Skewed distributions generated by the normal kernel. Statistics Probability Letters, 65, pp. 269-277, (2003).

[11] Pewsey, A. Problems of inference for Azzalini's skew-normal distribution. Journal of Applied Statistics, 27(7), pp. 859-870, (2000). 


\section{Osvaldo Venegas}

Departamento de Cs. Matemáticas y Físicas,

Facultad de Ingeniería,

Universidad Católica de Temuco,

Chile

e-mail : ovenegas@uct.cl

\section{Antonio I. Sanhueza}

Departamento de Matemática y Estadística,

Facultad de Ingeniería, Ciencias y Administración,

Universidad de La Frontera,

Chile

e-mail : asanhue@ufro.cl

and

\section{Héctor W. Gómez}

Departamento de Matemáticas,

Facultad de Ciencias Básicas,

Universidad de Antofagasta,

Chile

e-mail: hgomez@uantof.cl 